\title{
Contact Studies between Total Knee Replacement Components Developed Using Explicit Finite Elements Analysis
}

\author{
Lucian G. Gruionu ${ }^{1}$, Gabriel Gruionu ${ }^{2}$, Stefan Pastrama ${ }^{3}$, Nicolae Iliescu ${ }^{3}$, \\ and Taina Avramescu ${ }^{1}$ \\ ${ }^{1}$ University of Craiova, A.I.Cuza 13, Craiova, Romania \\ lucian.gruionudimst.ro \\ ${ }^{2}$ Indianapolis University Purdue University, 420 University Blvd. Indianapolis, IN, USA \\ ${ }^{3}$ University Politehnica of Bucharest, Splaiul Independentei 313, Bucharest, Romania
}

\begin{abstract}
A pneumatic simulator of the knee joint with five DOF was developed to determine the correlation between the kinematics of the knee joint, and the wear of the polyethylene componenent of a TKR prosthesis. A physical model of the knee joint with total knee replacement (TKR) was built by rapidprototyping based on CT images from a patient. A clinically-available prosthesis was mounted on the knee model. Using a video analysis system, and two force and contact pressure plates, the kinematics and kinetics data were recorded during normal walking of the patient. The quadriceps muscle force during movement was computed using the Anybody software. Joint loadings were generated by the simulator based on recorded and computed data. Using the video analysis system, the precise kinematics of the artificial joint from the simulator was recorded and used as input for an explicit dynamics FE analysis of the joint. The distribution of the contact stresses in the implant was computed during the walking cycle to analyze the prosthesis behavior. The results suggest that the combination of axial loading and anterior-posterior stress is responsible for the abrasive wear of the polyethylene component of the prosthesis.
\end{abstract}

\section{Introduction}

At present, the short life cycle of TKR represent a great concern for both orthopedic surgeons and prosthesis designers. Loosening of the tibial component is an important cause of TKR failure [1]. During the gait cycle, the forces developed in the knee have a cyclic pattern with values between 10 and $30 \mathrm{MPa}$, resulting in high stress values and wear of the artificial joint components. The mechanisms responsible for TKR wear are delamination, scratching, pitting and abrasion [1-3].

The present study used clinical investigation, engineering and computational methods: non-invasive imaging methods to collect data directly from the patient, a newly developed knee simulator to reproduce the physical forces acting on the joint during movement, and finite element analysis to integrate the clinical observations and experimental data and to compute the resulting stresses in the joint. The purpose of the study was to determine the correlation between the kinematics of the knee joint, and the stress distribution in the polyethylene componenent of the TKR during the gait cycle. 


\section{Materials and Methods}

The three-dimensional computational joint reconstruction (Fig. 1, a) was achieved from CT serial sections of the patient's leg and radiographic images of the joint with TKR. Images have been acquired in DICOM format and converted to JPEG using the MRIco software. The three-dimensional CAD model of the patient's knee joint was used to develop an artificial human knee by rapid prototyping using an Objet Eden $2603 \mathrm{D}$ printer. The femoral metallic components and the tibial metallic tray with polyethylene plate were mounted on the plastic components (Fig. 1, b) using medical cement. Five HBM LY strain gages were placed on both components of the joint for data recording during the simulation (Fig. 1,c).

A new 5DOF simulator for the knee joint was designed and developed in our labs to study the biomechanical changes in different pathological cases and to establish the efficacy of the type of osteotomy with different fixation systems and prostheses.
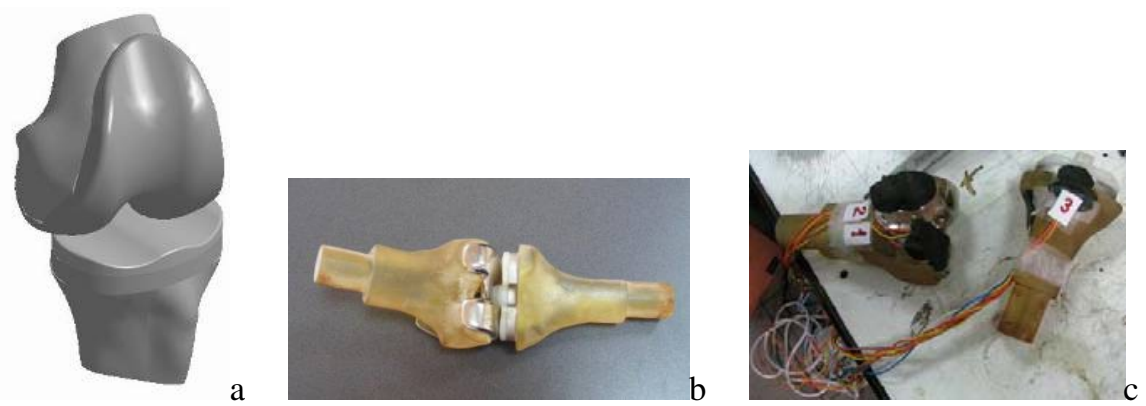

Fig. 1. Three-dimensional CAD models of the TKR joint of the patient (a), the prosthesis components and the artificial joint with implants mounted (b), the artificial femur and tibia with artificial prosthesis and strain markers (c)

The knee simulator is based on the general principles of the Purdue Knee Simulator with several novel patent-pending improvements. The new design allows an unconstrained flexion/extension motion between femur and tibia, with the hip and ankle joints attached to the frame (Fig. 2). The loads on the knee are: the simulated quadriceps muscle action (quadriceps load), and the applied external loads at the simulated hip (body weight) and ankle (vertical rotation torque and medial-lateral translation load) (Fig. 2, b). This particular design can simulate the natural behavior of the joint by varying the applied loads and articular geometry of the knee.

There are two DOF between the femur and simulator frame: vertical translation of the hip and flexion/extension/rotation relative to the translating hip sled. The hip sled is constrained by two vertical precision rails attached to the frame, and the load that simulates the body weight (max. $200 \mathrm{kgf}$ ) is provided by a pneumatic cylinder. The flexion-extension rotation $\left( \pm 65^{\circ}\right)$ is imposed through the quadriceps muscle simulated by an actuator fixed to the femur. The action of the quadriceps actuator cause an extension moment about the knee that extended the hip as well. The ankle sled provides four degrees of freedom between the tibia and the ground. A universal joint gives the 


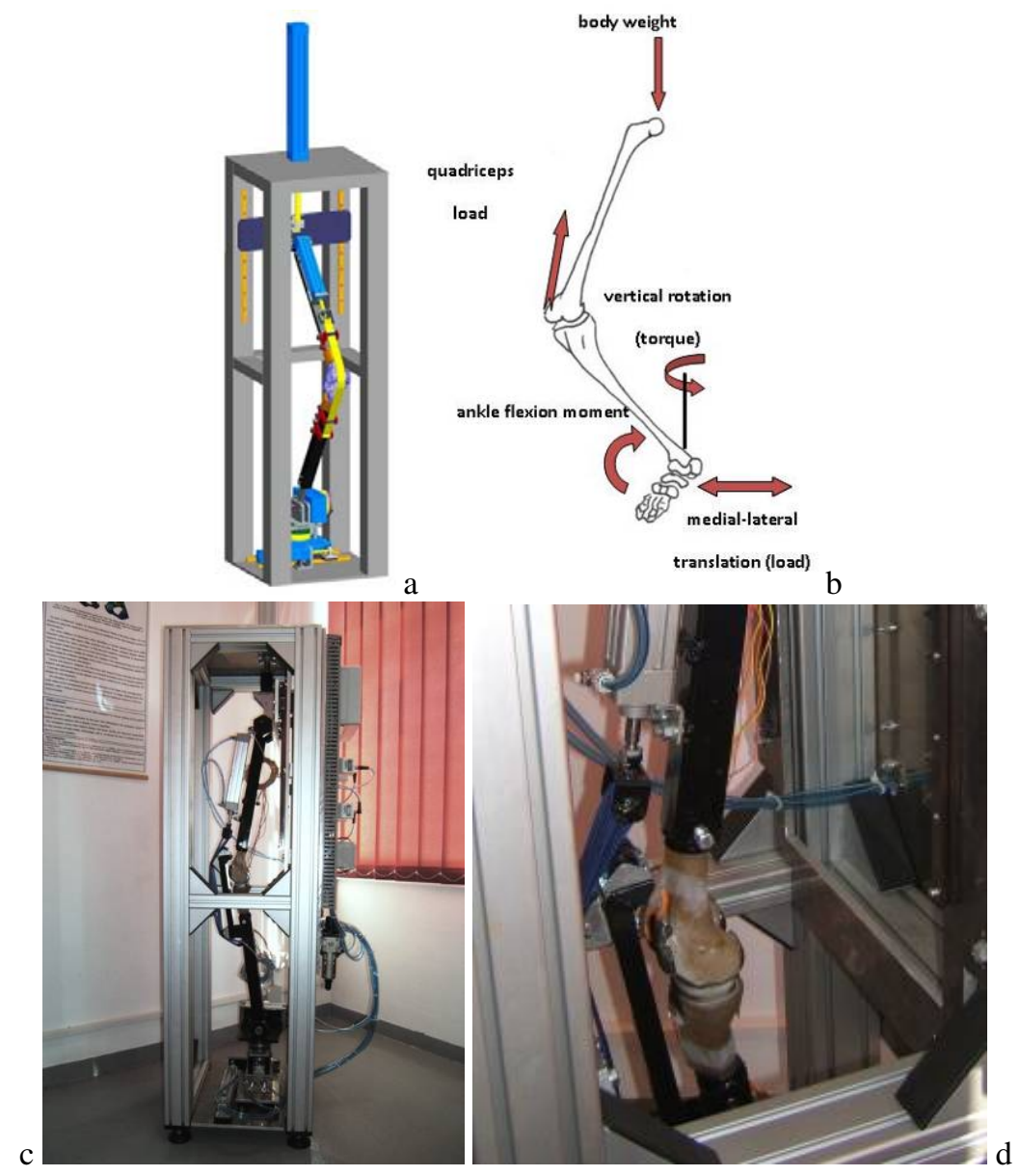

Fig. 2. The CAD design (a), resultant loads diagram on the joint (b), the newly developed knee simulator (c) and close-up of the knee joint region (d)

flexion-extension and adduction-abduction of the tibia at the ankle. The universal joint also rotate about a vertically oriented axis $\left( \pm 20^{\circ}\right)$. The ankle assembly is free to move on a medial/lateral direction $( \pm 7.5 \mathrm{~mm})$. The motion of the ankle sled is controlled by the adduction-abduction load actuator (max. 1000N).

The tibia is free to abduct/adduct. Its motion is dependent on the medial-lateral translation of the ankle sled since the femur is fixed to the frontal plane. Internalexternal rotation of the tibia is controlled by a rotational actuator (max. $45 \mathrm{Nm}$ ) in a vertical axis translating with respect to the ankle sled.

The ankle flexion moment induced by a rotational actuator (max. $55 \mathrm{Nm}$ ), applies a torque between the tibia and the ankle sled relative to the flexion-extension axis. The actuator translates in the medial-laterally direction, and rotates relative to the vertical axis of the tibia. 
Mechanical Testing of the Knee Joint. The testing was performed on an artificial knee implanted with a clinically-available prosthesis. The plastic femur and tibia were mounted on stainless steel tubes in the aluminum fixture. Both tubes include a threeaxis force and a torque sensor allowing a closed-loop feedback control.

The load profile on the ankle joint from the medial-lateral translation, and the torque movement were obtained directly from the patient using coupled AMTI/RSSCAN force/pressure plates. The values of the quadriceps load and ankle flexion moment during walking cycle were computed using the Anybody and Gait Model (Aalborg Univ.) software packages (Fig. 3). The patient lower member segments dimensions, body weight and foot center of pressure constitute the input data for the Anybody model.
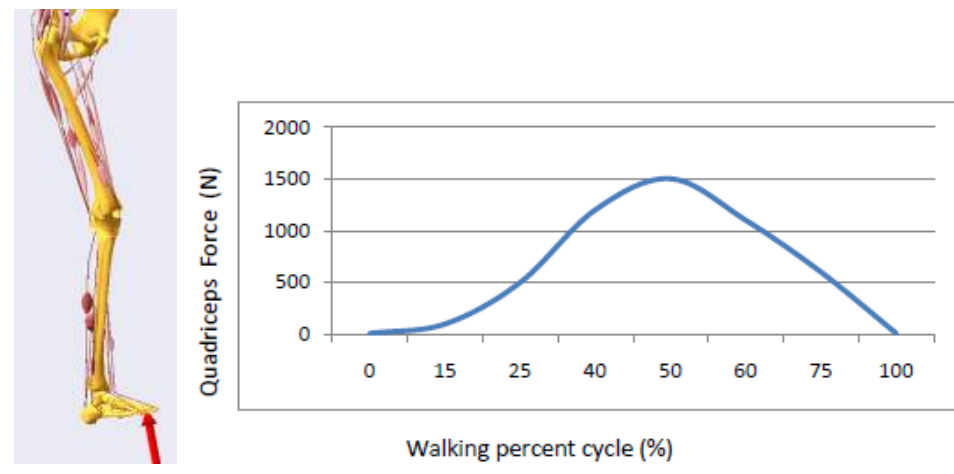

Walking percent cycle (\%)

Fig. 3. Anybody model and quadriceps muscle values $(\mathrm{N})$ during the walking cycle

The force generated by all pneumatic actuators were set to half of the measured values during the walking cycle to prevent damage to the artificial plastic model, which has a lower mechanical strength compared to the natural bone. The pressure values were also adjusted to account for differences in congruency between the synthetic and biological component surfaces during movement.

The movement of the joint in the simulator was recorded using two high speed (100f/s) Basler cameras and an image acquisition and analysis system, SIMI Motion (SIMI Reality Motion Systems GmbH). The kinematics data for the artificial joint have been obtained and the kinematics curves have been plotted (Fig. 4, a, b).

The finite element model of surface of the TKR prosthesis has been developed on the three-dimensional model using Ansys/LS-DYNA software (Fig. 4, c).

To decrease the computational burden and facilitate the analysis, the femoral implant and polyethylene tibial element were separated from the model.

The contact/impact conditions were simulated based on the assumption that at the time of contact the two surfaces have the same distortion speed in the direction of the impact. In this way, the impact was separated from the rest of the dynamic analysis.

The values (solutions) of the serial impulse equations were propagated over the impact, and generate initial conditions for the subsequent steps of the analysis. The erosion of the polyethylene component was estimated by calculating the quantity of material removed by friction for a given contact pressure. Experimentally, the degree of erosion can be assessed by weighing the polyethylene component after a large number of movement cycles and observing its microstructure under the microscope. 


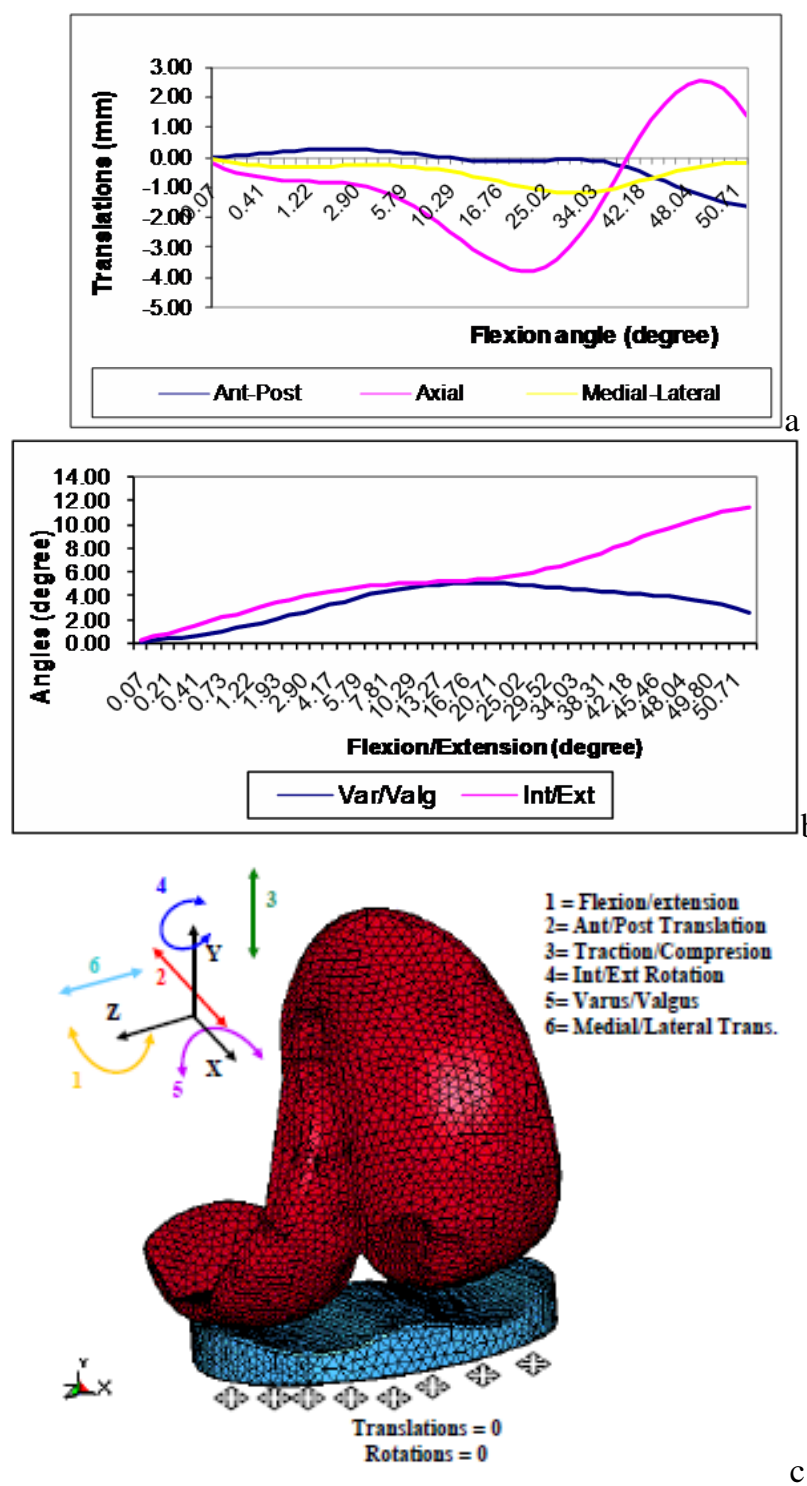

Fig. 4. The knee joint kinematics obtained with the simulator, and analyzed with the SIMI Motion software: translations (a) and rotations (b). The 3D finite elements model of the femoral component, tibial polyethylene and boundary conditions (c).

\section{Results}

The correlation between the TKR kinematics and tibial polyethylene loading was computed for two different ankle flexion moments, $15^{\circ}$ and $20^{\circ}$ flexion, corresponding to mid stance during the gait cycle (Fig. 5). 
LS-DYNA user input

$\min =-4.24301$, at elem\# 60201

$\min =-4.24301$
$\max =14.5845$

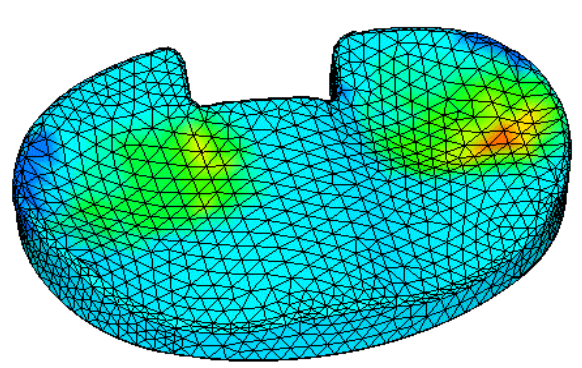

LS-DYNA user input

Contours of Pressure

$\mathrm{min}=-4.11434$, at elem\# 55904

$\max =12.8552$, at elem\# 57083

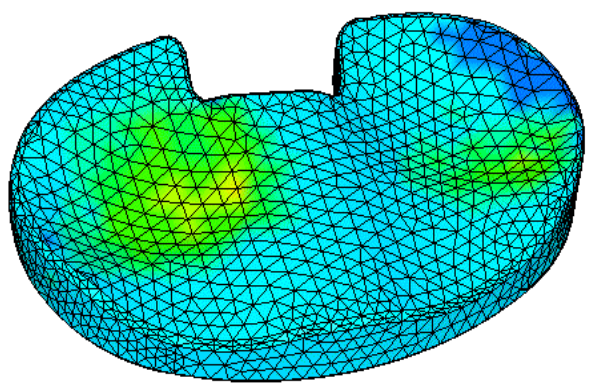

Fringe Levels

$1.458 \mathrm{e}+01$

$1.270 \mathrm{e}+01$

$1.082 \mathrm{e}+01$

$8.936 e+00$

$7.053 \mathrm{e}+00$

$5.171 \mathrm{e}+00$

$3.288 \mathrm{e}+00$

$1.405 e+00$

$-4.775 \mathrm{e}-01$

$-2.360 \mathrm{e}+00$

$-4.243 \mathrm{e}+00$

a

Fringe Levels

$1.286 \mathrm{e}+01$

$1.116 \mathrm{e}+01$

$9.461 \mathrm{e}+00$

$7.764 \mathrm{e}+00$

$6.067 \mathrm{e}+00$

$4.370 \mathrm{e}+00$

$2.673 \mathrm{e}+00$

9.765e-01

$-7.204 \mathrm{e}-01$

$-2.417 \mathrm{e}+00$

$-4.114 \mathrm{e}+00$

Fig. 5. Contact pressure (in MPa) for $15^{\circ}$ (a) and $20^{\circ}$ (b) flexion angles

Table 1. Contact pressure (in MPa), shear stress, anterior-posterior stress, axial stress, medial-lateral stress and peripheral stress

\begin{tabular}{|c|c|c|c|}
\hline & & $15^{\circ}$ of flexion & $20^{\circ}$ of flexion \\
\hline \multirow[t]{2}{*}{ Contact pression } & Internal compartment & 14,5 & 9,46 \\
\hline & External compartment & 8,9 & 7,76 \\
\hline \multirow[t]{2}{*}{ Shear stress } & Internal compartment & 3,2 & 2,97 \\
\hline & External compartment & 2,49 & 1,98 \\
\hline \multirow[t]{2}{*}{ A - P stress } & Internal compartment & 12,8 & 6,79 \\
\hline & External compartment & 8,4 & 5,21 \\
\hline \multirow[t]{2}{*}{ Axial loading } & Internal compartment & 14,01 & 9,29 \\
\hline & External compartment & 11,7 & 7,45 \\
\hline \multirow[t]{2}{*}{ M - L stress } & Internal compartment & 8,07 & 7,97 \\
\hline & External compartment & 8,07 & 4,11 \\
\hline \multirow[t]{2}{*}{ Peripheral loading } & Internal compartment & 7,05 & 4,3 \\
\hline & External compartment & 4,24 & 2,4 \\
\hline
\end{tabular}


Polyethylene loadings were highest for $15^{\circ}$ flexion corresponding to the mid stance of the gait cycle. For comparison we used a second position of $20^{\circ}$ flexion. For the first position $\left(15^{\circ}\right.$ flexion), the contact pressure in the medial compartment is 14.5 $\mathrm{MPa}$, and is correlated with axial loading (14.01 MPa). For the $20^{\circ}$ flexion, contact pressure is 9.46 $\mathrm{MPa}$ and the axial loading is 9.29 $\mathrm{MPa}$ (Table 1).

\section{Conclusions}

The present paper used interdisciplinary engineering methods to understand the clinical problem of the wear of the TKR prostheses. The novelty of the study comes from using a new knee joint simulator, image acquisition and interpretation techniques, but also numerical methods to simulate the inverse dynamics of the patient lower limb and finite elements models to determine the explicit dynamics of the stress and strain. The input data for the computational simulations was collected directly from the patient (joint geometry and magnitude of physical forces), and from experiments performed with the joint simulator. The output data resulted from computation can be verified using strain markers on the artificial joint in simulator.

The relationship between axial loading and anterior-posterior stress is responsible for the initiation of abrasive wear of polyethylene. The stress in this direction is maximal in the internal compartment for a $15^{\circ}$ flexion. The delamination of polyethylene can result from association at the same level of shear and axial forces.

The same pattern of stress distribution is observed in the external compartment but the values are lower. The difference between medial and lateral compartments is related to the presence of the various moments at the level of internal compartment in the unipodal weight bearing.

The presented simulator and the overall research methodology were designed for the study of the biomechanical behavior of bone components of the knee joint in different pathological cases and different types of movement. This system can also be used for fatigue and wear studies of some types of the prosthesis and osteotherapy devices.

The simulator can be used for research, prosthetics testing, and orthopedic surgeon training. Future studies using the knee simulator are needed to continue to determine and the regions where the physical forces produce wear and fatigue into the TKRimplant.

\section{References}

1. Nordin, M., Frankel, V.H.: Basic Biomechanics of the Musculoskeletal System, p. 400. Lippincott Williams \& Wilkins (2001)

2. De Lima, D.D., Chen, P.C., Colwell, C.W.: Polyethylene contact stresses, articular congruity, and knee alignment. Clin. Orthop., 232-238 (2001)

3. Perie, D., Hobatho, M.C.: In vivo determination of contact areas and pressure of the femorotibial joint using non-linear finite element analysis. Clin. Biomech. (Bristol, Avon) 13, 394-402 (1988)

4. Waldman, S.D., Bryant, J.T.: Dynamic contact stress and rolling resistance model for total knee arthroplasties. J. Biomech. Eng. 119, 254-260 (1997) 